\title{
Transition Temperatures of Thermotropic Liquid Crystals from the Local Binary Gray Level Cooccurrence Matrix
}

\author{
S. Sreehari Sastry, ${ }^{1}$ T. Vindhya Kumari, ${ }^{1}$ C. Nageswara Rao, ${ }^{1}$ K. Mallika, ${ }^{1}$ \\ S. Lakshminarayana, ${ }^{2}$ and Ha Sie Tiong ${ }^{3}$ \\ ${ }^{1}$ Department of Physics, Acharya Nagarjuna University, Nagarjuna Nagar, Andhra Pradesh 522510, India \\ ${ }^{2}$ Department of Electronics and Communication Engineering, K L University, Greenfields, Vaddeswaram, Guntur, \\ Andhra Pradesh 522002, India \\ ${ }^{3}$ Department of Chemical Science, Faculty of Science, Universiti Tunku Abdul Rahman and Jalan Universiti, Bandar Barat, \\ 31900 Kampar, Perak, Malaysia
}

Correspondence should be addressed to S. Sreehari Sastry, sreeharisastry@yahoo.com

Received 7 October 2012; Revised 19 November 2012; Accepted 20 November 2012

Academic Editor: Nigel Wilding

Copyright (c) 2012 S. Sreehari Sastry et al. This is an open access article distributed under the Creative Commons Attribution License, which permits unrestricted use, distribution, and reproduction in any medium, provided the original work is properly cited.

This paper presents a method which combines the statistical analysis with texture structural analysis called Local Binary Gray Level Cooccurrence Matrix (LBGLCM) to investigate the phase transition temperatures of thermotropic $p, n$-alkyloxy benzoic acid ( $n \mathrm{OBA}, n=4,6,8,10$ and 12) liquid crystals. Textures of the homeotropically aligned liquid crystal compounds are recorded as a function of temperature using polarizing optical microscope attached to the hot stage and high resolution camera. In this method, second-order statistical parameters (contrast, energy, homogeneity, and correlation) are extracted from the LBGLCM of the textures. The changes associated with the values of extracted parameters as a function of temperature are a helpful process to identify the phases and phase transition temperatures of the samples. Results obtained from this method have validity and are in good agreement with the literature.

\section{Introduction}

Thermotropic liquid crystals exhibit a variety of phases and phase transitions in the materials [1-3]. The structure of thermotropic liquid crystal phases is easily influenced by external conditions, for example, the properties of the surface of a microscope cover glass. In case of planar alignment of LC's the phases can possess complex structures and properties which are studied from several decades. In contrast, the investigation of liquid crystals as components in heterogeneous and its anchoring homeotropically is still in an early stage, especially when the more complex phases such as different smectic phases are considered. The understanding of textures and their characteristic features of thermotropic liquid crystal is the main focus of our studies to investigate the phase transitions [4].

There are several techniques used to identify the mesophases or phase transitions of liquid crystals, such as polarizing optical microscope (POM), differential scanning calorimetry (DSC), differential thermal analysis (DTA), and $\mathrm{X}$-ray diffraction. POM is one of the essential tools for the characterization of newly synthesized mesogenic materials, together with DSC, DTA, and X-ray investigations. While DSC and DTA are easily and quickly carried out methods, they merely provide information on phase transition temperatures, heat of transitions, heat capacity of various phases, and the order of the transitions. X-ray investigations for determination of the phase type have to be performed on macroscopically well-oriented samples which is often time consuming and sometimes hard to realize. However, precise phase identification could not easily be made by DSC, DTA, and X-ray investigation approaches. Polarizing microscopy on the other hand can provide a determination of both phase transition temperatures and phase type. This is done by temperature dependent texture investigations 
between crossed polarizers. Changes in the characteristics of the textural patterns that occurred while passing a phase transition reveal the type of phase presence $[5,6]$. Keeping this in mind, some researchers proposed a liquid crystal texture image processing and analysis methodology to investigate the phase transitions of liquid crystals. They used the statistical parameters of the first and second kinds to identify the phase transitions temperatures of liquid crystals [7-11]. However there exists coherence length diagram for the analysis [12] of liquid crystal textures which involves the more numbers of pixels. This coherence length analysis is useful to characterize the irregular textures, subphases, and defects. Coherence length analysis ignores the boundaries of the textures and the complexity involved in required parameters such as saturation point; threshold and directions for the calculation of coherence lengths are high. The statistical parameters of the first and second kind extract textural features based on one or two pixel intensity values which ignore the spatial relationship between the texture patterns. Therefore it is reasonable to propose a new method that combines the statistical analysis method with texture structural method which provides the information regarding the texture patterns and their strengths. Gray Level Cooccurrence Matrix of Local Binary Pattern (LBP) image extracts the features based on local neighbourhood of image which contains all spatial and texture feature information of different patterns. Extraction of parameters from the LBGLCM of textures is a helpful process to identify the textural features and it changes efficiently in order to investigate the phase transitions of the samples. In this communication a texture structural analysis method called Local Binary Gray Level Cooccurrence Matrix (LBGLCM) is proposed to investigate the phase transition temperatures of $p, n$-alkyloxy benzoic acids ( $n \mathrm{OBA}, n=4,6,8,10$ and 12 ) and results are compared with the literature.

\section{Experiment}

Thermotropic liquid crystals of even homologous series, $p, n$-Alkyloxybenzoic acid ( $n \mathrm{OBA}$, where $n=4,6,8,10$, and 12), are obtained from the Frinton laboratories, Inc., USA. The homeotropic alignment $\left(90^{\circ}\right.$ alignment $)$ of ITO coated cell having area of $5 \mathrm{~mm} \times 5 \mathrm{~mm}$ with $6 \mu \mathrm{m}$ spacing (tolerance is $\pm 0.2 \mu \mathrm{m}$ ) are obtained from Instec Inc., USA. Temperature dependent textural investigations are carried out by Meopta optical polarizing microscope attached to hot stage as described by Gray [13] and high resolution Canon camera. Mesogens are injected in these cells by heating the sample in its isotropic state through capillary action.

A high resolution Canon color camera is used for recording the observations. The colour image or texture recorded by the camera has a resolution of $3888 \times 2592$ pixels which represents the 24-bit true colour pixel tone that ranges from 0 to 255 in R, G, and B colours. In the present work, the translated gray scale image is used for analysis. The size of the image is selected to be 256 . The program has been coded using MATLAB software (implemented on P4 1.6 GHz with
2 GB RAM) for computational analysis of textures which is an efficient tool for computation [14].

\section{Texture Features Extraction from the Local Binary Gray Level Cooccurrence Matrix (LBGLCM)}

A new approach based on the idea of Local Binary Pattern (LBP) operator and Gray Level Cooccurrence Matrix (GLCM) is proposed. Firstly, we have to apply the local binary pattern (LBP) operator on liquid crystal textures generating the LBP image. Secondly, the GLCM of the LBP images has to construct and finally we have to extract the second-order statistical parameters (contrast, energy, homogeneity, and correlation) that are extracted from the Local Binary Gray Level Cooccurrence Matrix of textures.

3.1. Local Binary Pattern Operator. The local binary pattern (LBP) operator is defined as a gray scale invariant texture measure, derived from a general definition of texture in a local neighbourhood. It can be seen as a unifying approach to the traditionally divergent statistical and structural models of textural analysis. Perhaps the most important property of the LBP operator in real-world applications is its invariance against monotonic gray level change. Another equally important thing is its computational simplicity, which makes it possible to analyze the images in challenging real-time settings [15-17]. The LBP operator was originally designed for texture description. This concept was developed by Ojala et al. [18], attempts to decompose the texture into small units where the texture features are defined by the distribution of the LBP values calculated for each pixel in the image. The LBP texture unit is calculated in a $3 \times 3$ square neighbourhood by applying a simple threshold operation with respect to the central pixels as illustrated in (1):

$$
\begin{gathered}
T=\left\{t\left(g_{0}-g_{c}\right), \ldots, t\left(g_{P-1}-g_{c}\right)\right\}, \\
t(x)= \begin{cases}1 & x \geq 0 \\
0 & x<0\end{cases}
\end{gathered}
$$

where $T$ is the texture unit, $g_{c}$ is the gray level value of the central pixel, $g_{P}$ are the gray values of the pixels adjacent to the central pixel in the $3 \times 3$ neighbourhood, $P$ defines the number of pixels in the $3 \times 3$ neighbourhood, and function $t$ defines the threshold operation. For a $3 \times 3$ neighbourhood the value of $P$ is 8 . To encompass the spatial arrangement of the pixels in the $3 \times 3$ neighbourhood, the LBP value for the tested (central) pixel is calculated using the following relationship:

$$
\mathrm{LBP}=\sum_{i=0}^{P-1} t\left(g_{i}-g_{c}\right) * 2^{i},
$$

where $t\left(g_{i}-g_{c}\right)$ is the value of the thresholding operation illustrated in (1). The LBP values calculated using (2) is in the range [0-255]. Figure 1 is an illustration of the basic LBP operator. It concerns the spatial relationship 


\begin{tabular}{|c|c|c|c|c|c|c|c|c|c|c|c|}
\hline 6 & 5 & 4 & \multirow{3}{*}{ Threshold } & 1 & 1 & & & & & & \multirow{3}{*}{$\begin{array}{l}\text { Pattern: } 01100111 \\
\text { LBP: } 64+32+4+2+1=103\end{array}$} \\
\hline 3 & 3 & 2 & & 0 & & 0 & & & & 8 & \\
\hline 5 & 6 & 1 & & 1 & 1 & 0 & & 64 & 32 & 16 & \\
\hline
\end{tabular}

Figure 1: Illustration of LBP operator.

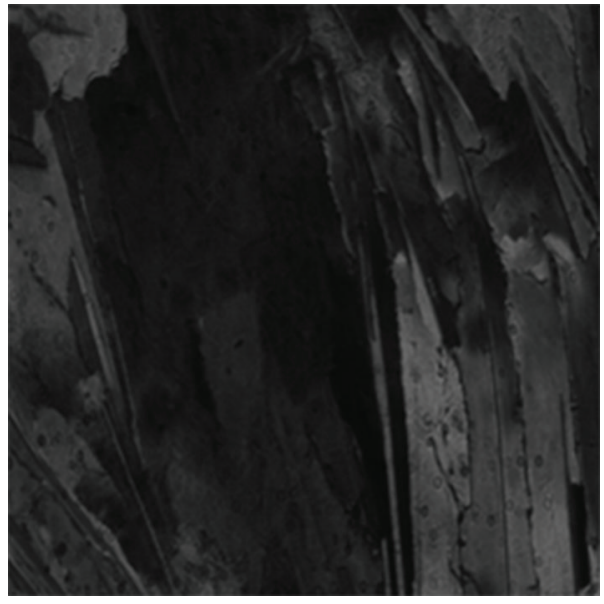

(a)

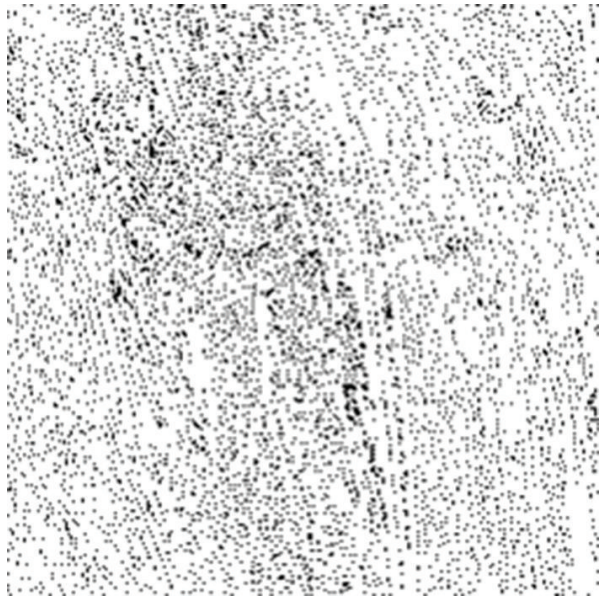

(b)

Figure 2: (a) Gray scale image. (b) The LBP image.

between patterns while ignoring the magnitude of gray level differences. And also invariant against gray scale shifts, that is, the texture features are invariant when all of the pixels in the neighbourhood are plus or minus a value at the same time, so the sign of differences can also be ignored [19]. For example, the gray scale image and the processed image from LBP operator are shown in Figure 2.

3.2. Gray Level Cooccurrence Matrix (GLCM). The Gray Level Cooccurrence Matrix (GLCM) is a widely used texture analysis method especially for stochastic textures. It enhances the details of image and gives the interpretation. The GLCM is a tabulation of how often different combinations of pixel brightness values (gray levels) occur in an image. The GLCM indicates the frequency occurrence of pixel pairs. From this principal, it is used to compute the relationships of pixel intensity to the intensity of its neighbouring pixels which are based on hypothesis that the same gary level configuration is repeated in a texture [20-22].

The GLCM is defined with respect to given row, column. A GLCM element $(i, j)$ denoted by $C_{i j}$ represents the number of times a point having gray level $j$ occurs relative to a point having gray level $i$. This was explained with example shown in Figure 11. In Figure 11, each element of Gray Level Cooccurrence Matrix represents the probability of occurrence of pixel pair. In (Figure 11) 20 and right-hand neighbor 10 occur, that is, $(20,10)$ at two times. Therefore element of GLCM represents the probability of occurrence of pixel pair, that is, 2 .

3.3. Feature Extraction from the LBGLCM. The traditional GLCM extracts the features based on pixel and its next neighbour pixel while ignoring the spatial relationship of different local texture patterns in the image [11]. GLCM of the LBP image extracts the features based on local neighbourhood which contains all spatial and texture feature information of different patterns. We can extract the secondorder statistical parameters: contrast, energy, homogeneity, and correlation from the LBGLCM and they will identify the textural features and their change, efficiently.

An image of $\operatorname{LBGLCM}(i, j)$ of size $m$-by- $n$ is a twodimensional function and it is composed of $m$ pixels in the vertical direction and $n$ pixels in the horizontal direction, $i, j$ are horizontal and vertical coordinates of the image. The total number of pixels in the image is $m * n=N, 0 \leq i \leq m, 0 \leq$ $j \leq n$. The defined second-order statistical parameters are as follows.

(i) Contrast. Intensity contrasts between different textural patterns are measured by the contrast of texture. The value of contrast is low if the gray levels of each textural patterns are similar.

Contrast is as follows:

$$
\sum_{i=1}^{m} \sum_{j=1}^{n}(i-j)^{2} \operatorname{LBGLCM}(i, j)
$$

(ii) Energy. Energy measures the textural uniformity. Maximum energy of the texture or image occurs when the gray level distribution of a given image is either constant or a periodic uniform.

Energy is as follows:

$$
\sum_{i=1}^{m} \sum_{j=1}^{n}(\operatorname{LBGLCM}(i, j))^{2}
$$




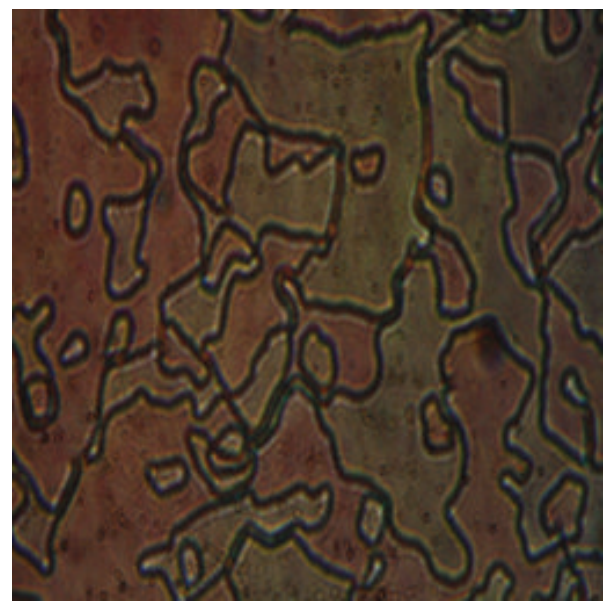

(a)

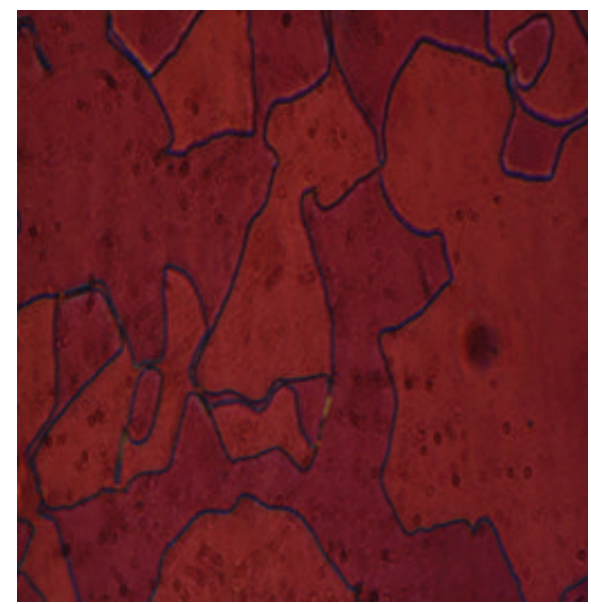

(b)

Figure 3: Nematic textures of (a) $4 \mathrm{OBA}$ at temperature $161^{\circ} \mathrm{C}$ and (b) $6 \mathrm{OBA}$ at temperature $150^{\circ} \mathrm{C}$.

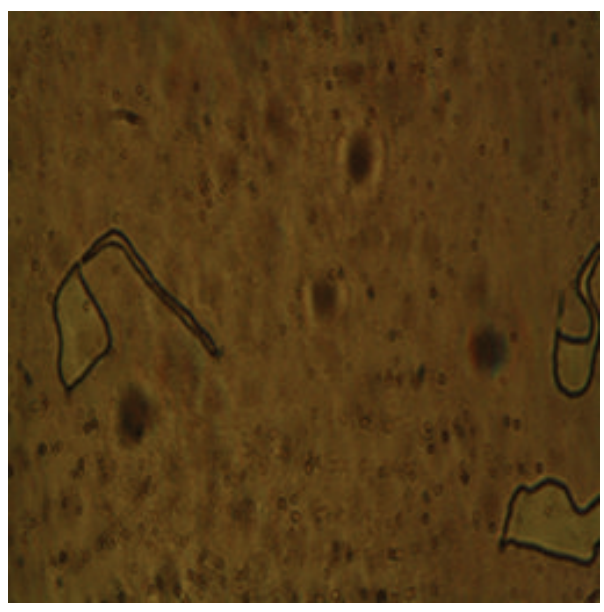

(a)

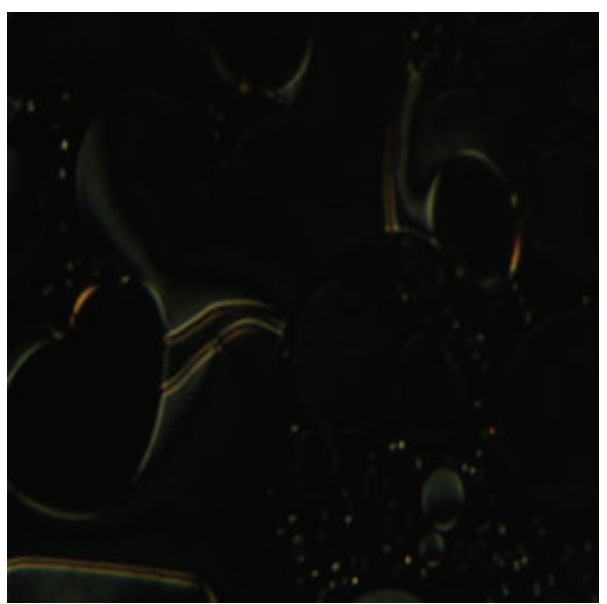

(c)

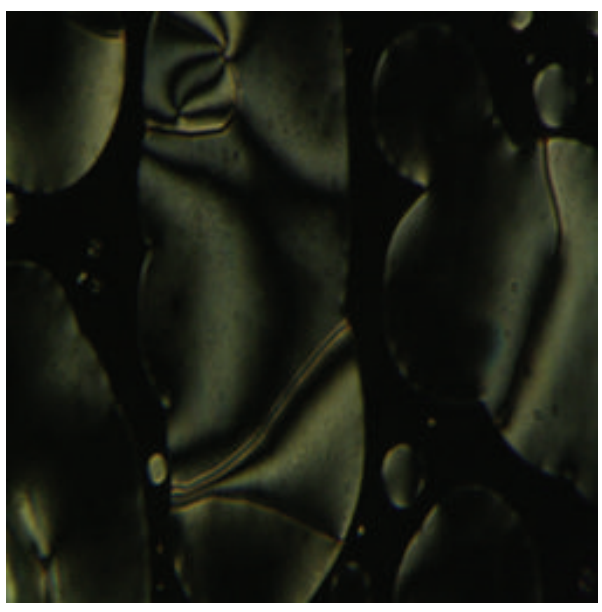

(b)

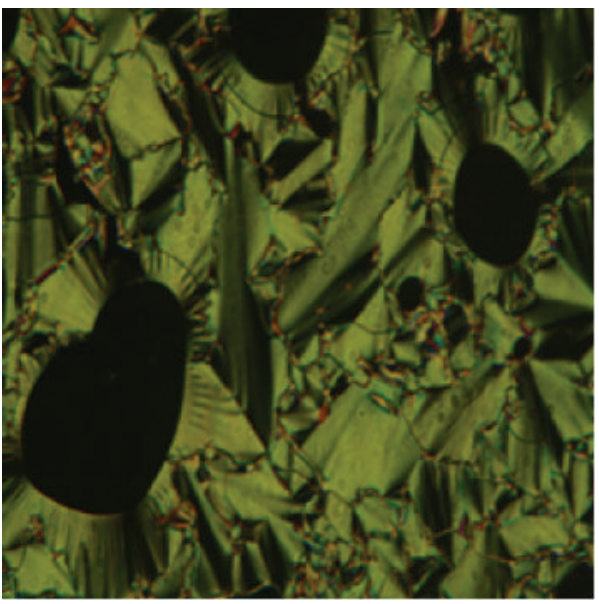

(d)

Figure 4: (a) Nematic texture of $8 \mathrm{OBA}$ at temperature $146.5^{\circ} \mathrm{C}$, (b) nematic texture $10 \mathrm{OBA}$ at temperature $123.4^{\circ} \mathrm{C}$, (c) nematic texture of $12 \mathrm{OBA}$ at temperature $135^{\circ} \mathrm{C}$ and (d) smectic texture of $12 \mathrm{OBA}$ at temperature $100^{\circ} \mathrm{C}$. 


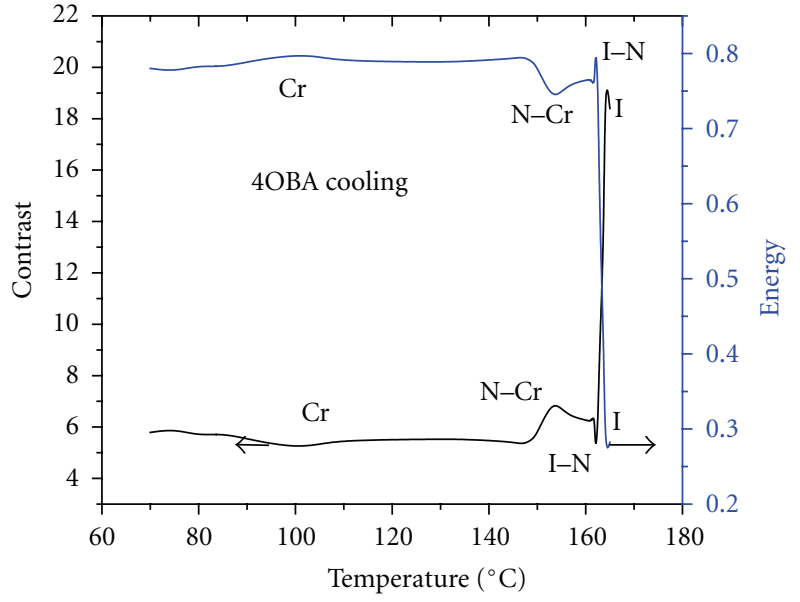

(a)

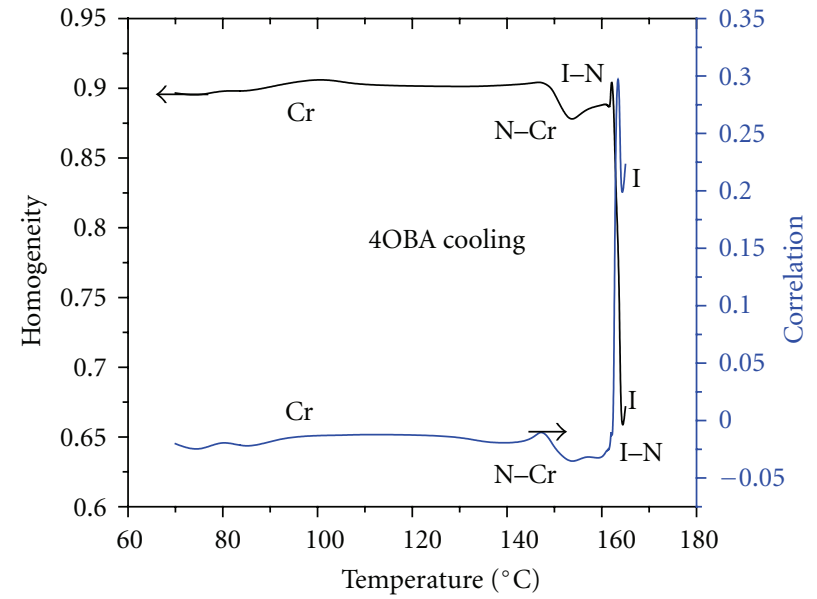

(b)

FIgURE 5: Computed parameters from the textures of 4OBA as a function of temperature where the phase transitions are indicated. (a) Contrast and energy; (b) homogeneity and correlation. Cr: Crystal, SmC: SmecticC, N: Nematic, and I: Isotropic.

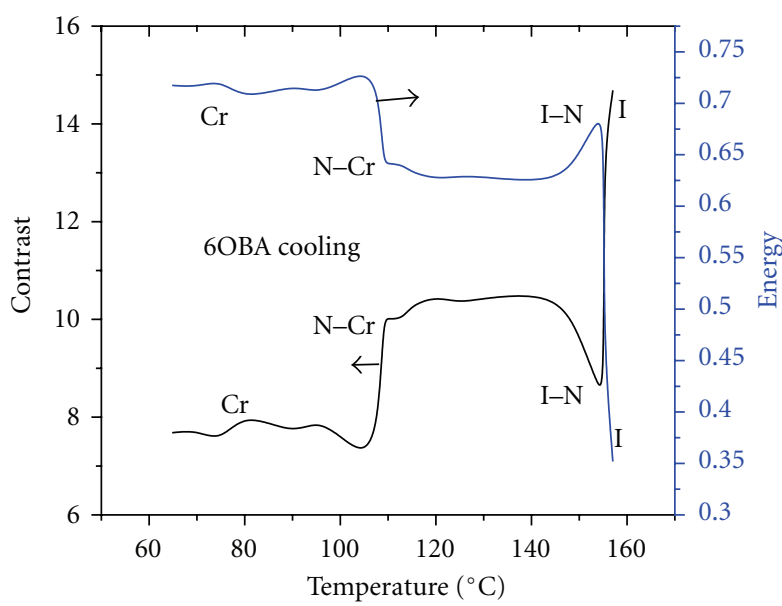

(a)

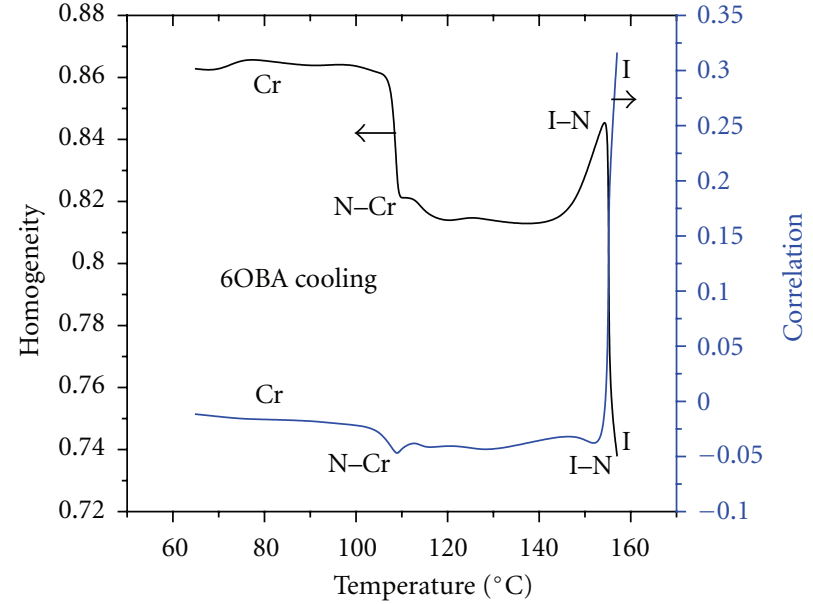

(b)

Figure 6: Computed parameters from the textures of 6OBA as a function of temperature where the phase transitions are indicated. (a) Contrast and energy; (b) homogeneity and correlation.

(iii) Homogeneity. Homogeneity measures the closeness of the distribution of gray values in the image. Homogeneous texture will contain only a limited range of gray levels, giving a LBGLCM with only a few values but relatively high probability.

Homogeneity:

$$
\sum_{i=1}^{m} \sum_{j=1}^{n} \frac{\operatorname{LBGLCM}(i, j)}{1+|i-j|}
$$

(iv) Correlation. Correlation is a measure of linear dependence between the textural patterns at specified positions relative to each other. Correlation is uncorrelated with energy, contrast, and homogeneity.
Correlation is as follows:

$$
\sum_{i=1}^{m} \sum_{j=1}^{n} \frac{\{i \times j\} \times \operatorname{LBGLCM}(i, j)-\left\{\mu_{x} \times \mu_{y}\right\}}{\sigma_{x} \times \sigma_{y}},
$$

where $\mu_{x}, \mu_{y}$, and $\sigma_{x}, \sigma_{y}$ are the mean and standard deviations of probability matrix $P$ along row-wise $\left(P_{x}\right)$ and column-wise $\left(P_{y}\right)$.

\section{Results and Discussions}

$n$ OBA $(n=4,6)$ exhibit the nematic phase and textures of those two samples are shown in Figure 3. The compounds $n$ OBA ( $n=8,10$ and 12 ) show both nematic, smecticC phases and textures of samples are shown in Figure 4. For the homologues series with $n=8,10$, and 12 containing 


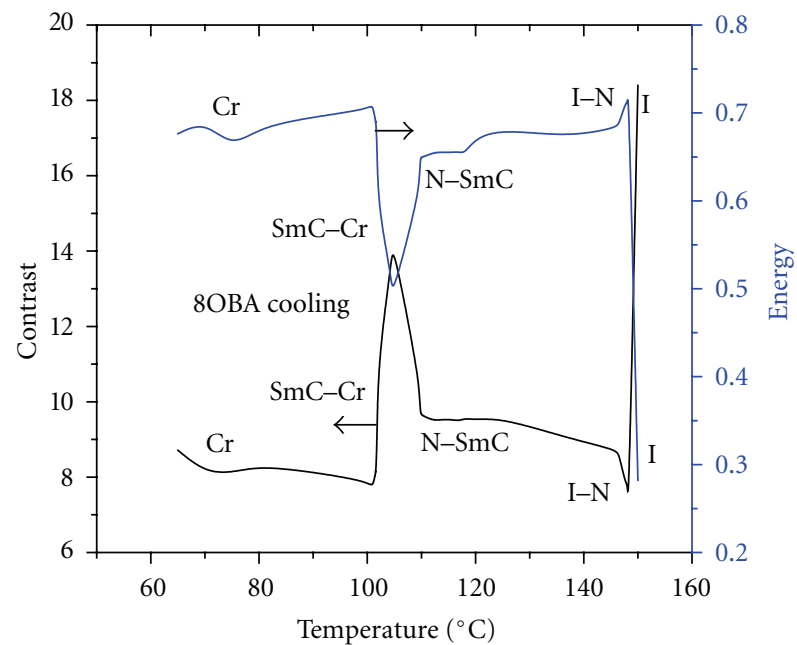

(a)

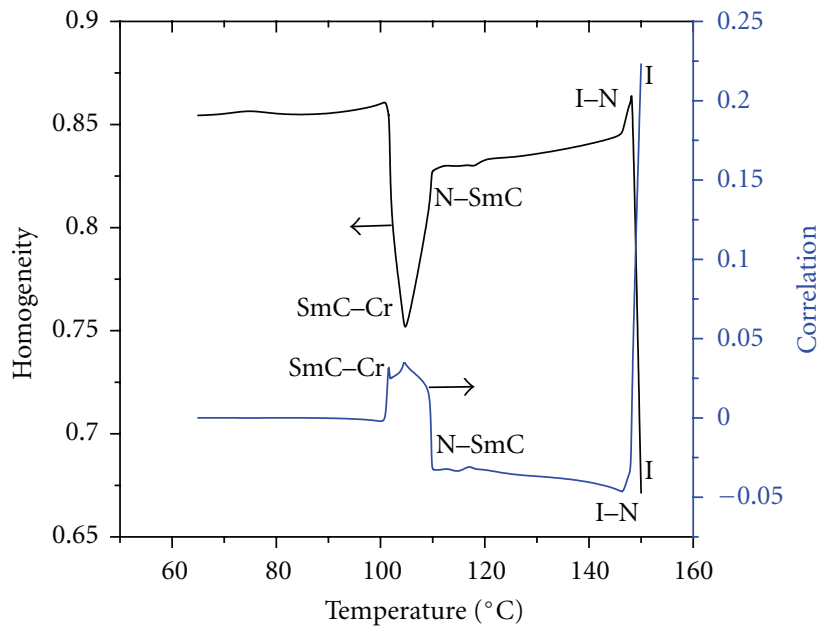

(b)

Figure 7: Computed parameters from the textures of 8OBA as a function of temperature where the phase transitions are indicated. (a) Contrast and energy; (b) homogeneity and correlation.

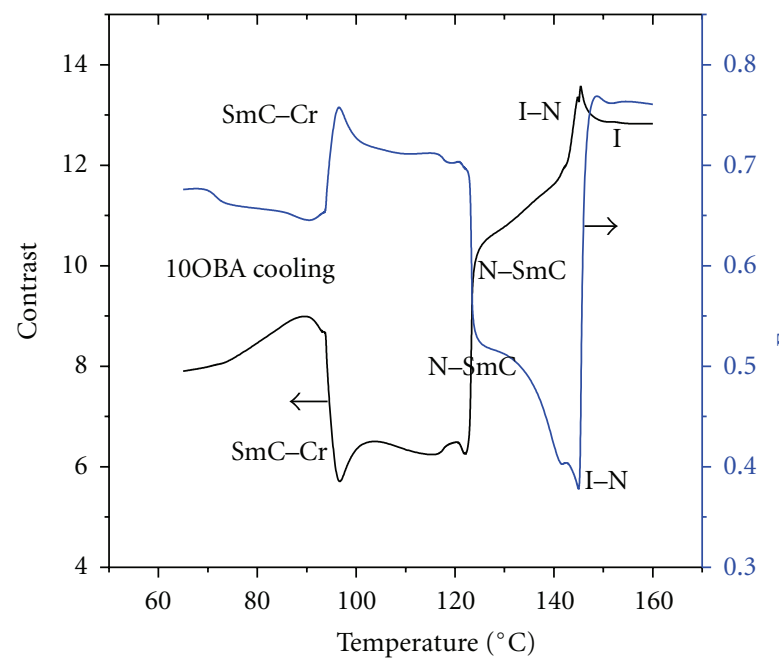

(a)

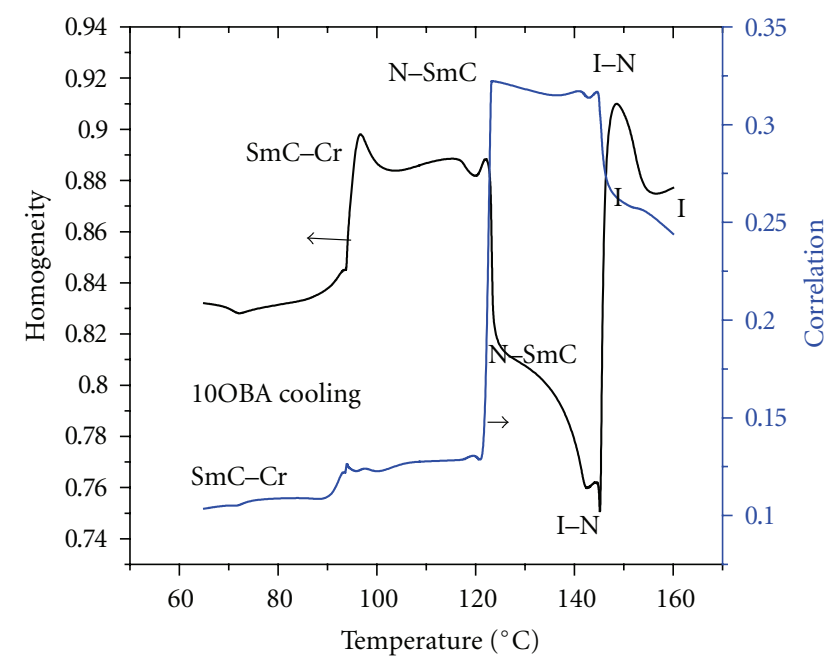

(b)

FIGURE 8: Computed parameters from the textures of 10OBA as a function of temperature where the phase transitions are indicated. (a) Contrast and energy; (b) homogeneity and correlation.

TABLE 1: Textures and phase transition temperatures of homeotropically aligned $p, n$-alkyloxybenzoic acid mesogens $(n=4,6,8,10$, and $12)$.

\begin{tabular}{lccc}
\hline Compound $n$ OBA where $n=$ & Phases & \multicolumn{2}{c}{$\begin{array}{c}\text { Phase transition temperatures }\left({ }^{\circ} \mathrm{C}\right) \\
\text { Present method }\end{array}$} \\
\hline 4 & & Literature [23] & $160-147$ \\
6 & $\mathrm{I}-\mathrm{N}-\mathrm{Cr}$ & $154-106$ & $163.7-149$ \\
8 & $\mathrm{I}-\mathrm{N}-\mathrm{Cr}$ & $147-108-101$ & $155.3-108$ \\
10 & $\mathrm{I}-\mathrm{N}-\mathrm{SmC}-\mathrm{Cr}$ & $142-122-97$ & $148.3-109.8-102.1$ \\
12 & $\mathrm{I}-\mathrm{N}-\mathrm{SmC}-\mathrm{Cr}$ & $137-129-95$ & $145.4-123.7-94$ \\
\hline
\end{tabular}

SmC: SmecticC; N: Nematic; Cr: Crysta; I: Isotropic. 


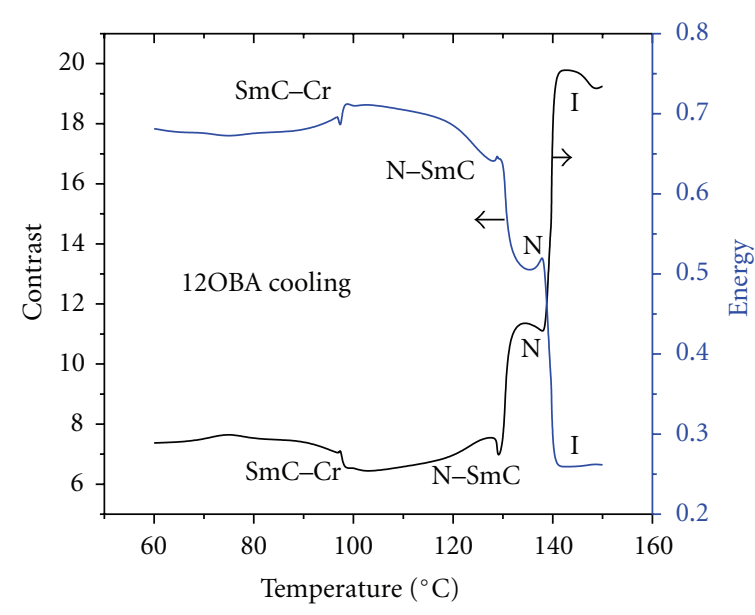

(a)

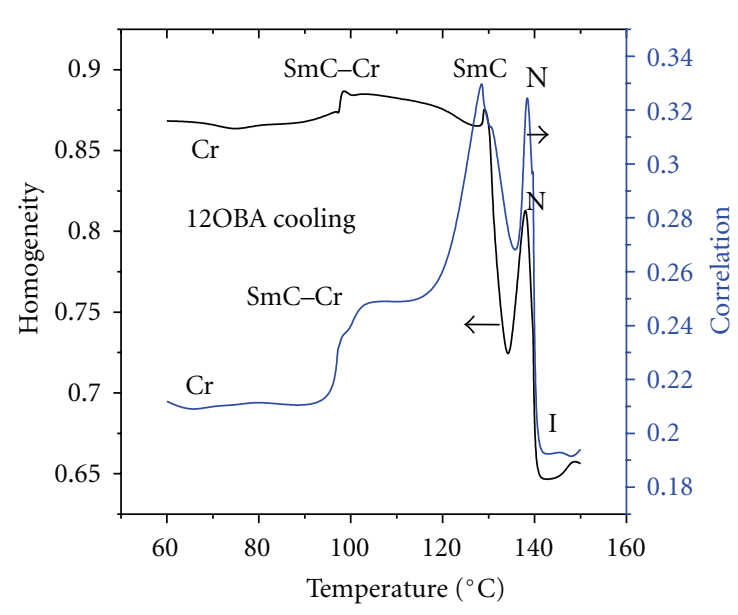

(b)

Figure 9: Computed parameters from the textures of 12OBA as a function of temperature where the phase transitions are indicated. (a) Contrast and energy; (b) homogeneity and correlation. Cr: Crystal, SmC: SmecticC, N: Nematic, I: Isotropic.

larger alkyloxy chain shows smecticC mesomorphism with broader mesomorphic range and chain length is important for the stabilization of the mesophases compared to series where $n=4,6$.

LBP of each temperature recording of the texture is computed using (1) and (2). After construction of GLCM of the LBP image, the second-order statistical parameters, contrast, energy, homogeneity, and correlation are computed from (3)-(6). At each temperature recording of the liquid crystal sample, the parameters are extracted from the textures and extracted parameters of the textures as a function of temperature that gives the phase transition of liquid crystals. The results obtained from this methodology are shown in Figures 5, 6, 7, 8 and 9.

The plots are drawn for parameters values of the $p, n$ alkyloxy benzoic acid textures as a function of temperature. The plots shown in Figures 5 to 9 reveal that, the parameter value remains approximately constant when there is no change in the textural features like isotropic phase or solid phase, once the phase transformation takes place from isotropic phase to the liquid crystal phase or liquid crystal phase to the solid phase reappearance of textural pattern or changes in textural features at specific temperature brings the abrupt variations in the parameter curves. In general, as $s$ function of temperature the changes in the characteristics of the textural patterns occurred while passing a phase transition.Therefore, the temperature corresponding to abrupt changes in the curve represents the transition temperature of sample. This implies that present methodology is a helpful process to investigate the phase transitions of liquid crystals. As a representative case, the plots are drawn for cooling cycles, to avoid the paramorphotic defects. On cooling, in between crossed polarizers the sample executes the different textures with respect to the different phases as a function of temperature. In all plots, the behaviors of the parameters are different for different compounds which depend on the phase transformation and thermal stability of the phase.
Transition temperatures of given samples are compared with the literature [23] which are shown in Table 1.

In DSC thermogram, the abrupt changes in the enthalpy and heat flow values are useful parameters to identify the transition temperatures of the samples and are shown in Figure 10. Liquid crystal texture analysis in conjunction with POM is a process in which the abrupt changes in textural feature values computed from the textures as a function of temperatures are useful to identify the transition temperatures of the samples. Both processes are correlated to each other in abrupt changes of their measured parameter values.

Although, in DSC the change of enthalpy values for the second-order transitions was relatively small and is not observable like first-order transitions [2]. These kinds of transitions are observable using traditional and LBGLCM statistical analysis. Computed parameter values associated with the changes textural feature values at the transition are useful to identify this kind of transitions. While present methodology is invariant against the gray scale changes causes the changes in the parameter values at the phase transformations are small compared to the traditional GLCM. The knowledge of traditional statistical and LBGLCM textural analysis methods in conjunction with POM is a more helpful process to identify second-order transitions and we have considered a future work for newly synthesized compounds.

\section{Conclusion}

It is concluded that the present method is successful in identifying the phase transition temperatures of thermotropic liquid crystals $p, n$-alkyloxy benzoic acids of even series using statistical analysis with texture structural analysis called Local Binary Gray Level Cooccurrence Matrix (LBGLCM). Computational simplicity of the present methodology makes it possible to analyze the textural features in real-time settings. 


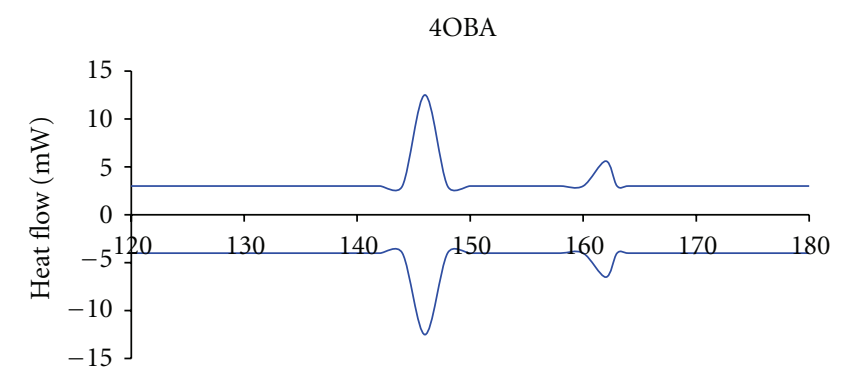

Temperature $\left({ }^{\circ} \mathrm{C}\right)$

(a)

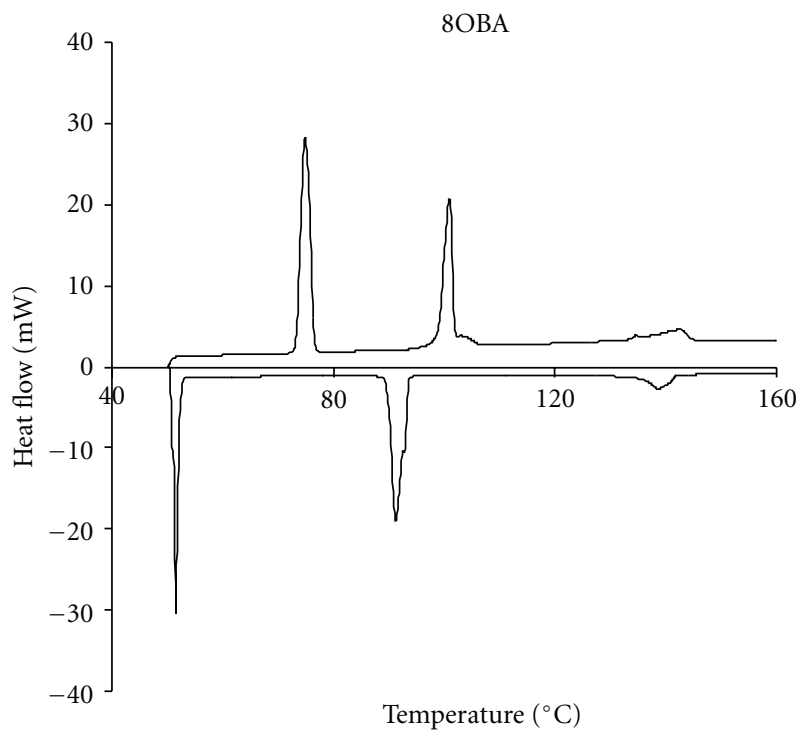

(c)

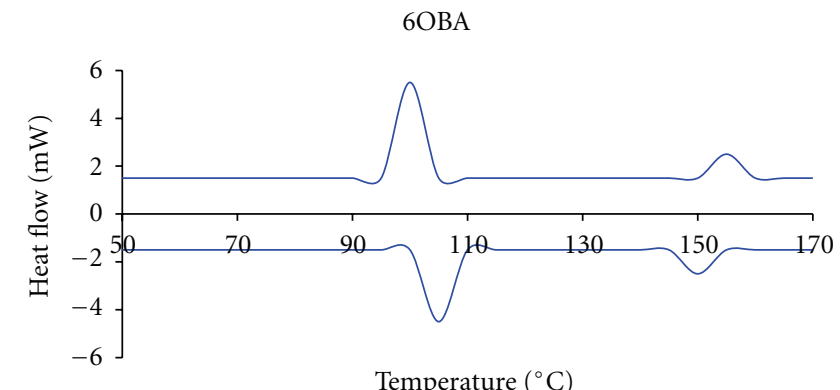

(b)

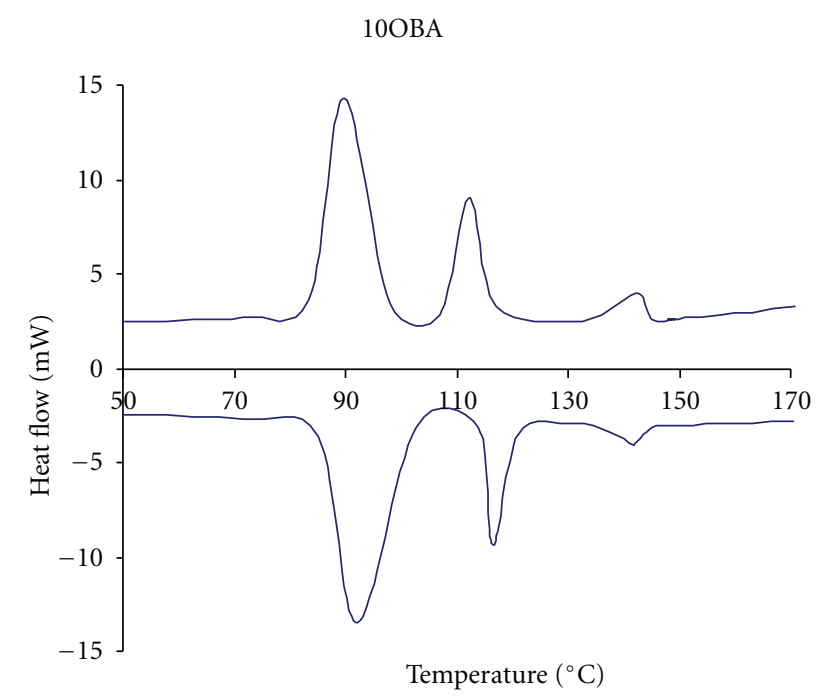

(d)

12OBA

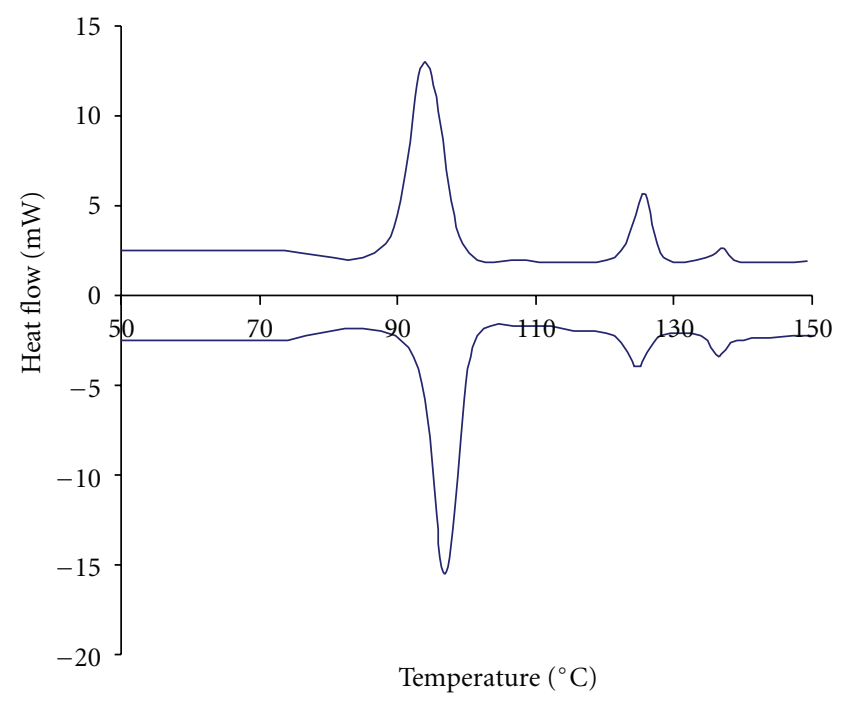

(e)

FIgURE 10: DSC thermograms of $p, n$-alkyloxybenzoic acid mesogens, (a) $n=4$, (b) $n=6$, (c) $n=8$, (d) $n=10$, and (e) $n=12$. 


\begin{tabular}{|c|c|c|c|c|}
\multicolumn{5}{c}{ Image } \\
\hline 20 & 5 & 10 & 5 & 5 \\
\hline 5 & 20 & 10 & 5 & 20 \\
\hline 10 & 5 & 5 & 10 & 5 \\
\hline 5 & 20 & 20 & 10 & 5 \\
\hline 10 & 5 & 10 & 10 & 5 \\
\hline
\end{tabular}

(a)

\begin{tabular}{|c|c|c|c|}
\multicolumn{5}{|c}{ GLCM of image } \\
\cline { 1 - 2 }$i / j$ & 5 & 10 & 20 \\
\cline { 1 - 1 } 5 & 2 & 3 & 3 \\
\cline { 1 - 1 } 10 & 7 & 1 & 0 \\
\cline { 1 - 1 } 20 & \multirow{2}{*n}{1} & 2 & 1 \\
\hline
\end{tabular}

(b)

Figure 11

\section{Acknowledgments}

The authors gratefully acknowledge the project no. 3412/2008 (SR), dated December 30, 2008 of UGC, UGC DRS LEVEL III program no. F.530/1/DRS/2009 (SAP-I), dated February 09, 2009, and DST FIST program no. DST/FST/ PSI-002/2011 dated December 20, 2011, New Delhi, to the Department of Physics, ANU for providing financial assistance.

\section{References}

[1] N. V. Madhusudana, "Recent advances in thermotropic liquid crystals," Current Science, vol. 80, no. 8, pp. 1018-1025, 2001.

[2] S. Chandrasekhar, Liquid Crystals, Cambridge University Press, Cambridge, UK, 1992.

[3] P. G. de Gennes and J. Prost, The Physics of Liquid Crystals, Clarendon Press, Oxford, UK, 1993.

[4] S. Sreehari Sastry, S. Kumar, T. Vindhya Kumari, K. Mallika, B. G. Sankara Rao, and H. S. Tiong, "Liquid crystal parameters through image analysis," Liquid Crystals, vol. 39, no. 12, pp. 1527-1537, 2012.

[5] S. Singh, Liquid Crystals Fundamentals, World Scientific, Singapore, 2002.

[6] D. Demus and L. Richter, Textures of Liquid Crystals, Chemie, New York, NY, USA, 1978.

[7] B. Montrucchio, A. Sparavigna, and A. Strigazzi, "A new image processing method for enhancing the detection sensitivity of smooth transitions in liquid crystals," Liquid Crystals, vol. 24, no. 6, pp. 841-852, 1998.

[8] B. Montrucchio, A. Sparavigna, S. I. Torgova, and A. Strigazzi, "A novel order transition inside the nematic phase of trans4-hexylcyclohexane-1-carboxylic acid discovered by image processing," Liquid Crystals, vol. 25, no. 5, pp. 613-620, 1998.

[9] A. Sparavigna, A. Mello, and B. Montrucchio, "Texture transitions in the liquid crystalline alkyloxybenzoic acid 6OBAC," Phase Transitions, vol. 79, no. 4-5, pp. 293-303, 2006.

[10] J. Eccher, A. R. Sampaio, R. C. Viscovini et al., "Image processing as a tool for phase transitions identification," Journal of Molecular Liquids, vol. 153, no. 2-3, pp. 162-166, 2010.

[11] S. Sreehari Sastry, K. Mallika, B. G. Sankara Rao, S. Tiong Ha, and S. Lakshminarayana, "Identification of phase transition temperatures by statistical image analysis," Liquid Crystals, vol. 39, no. 6, pp. 695-700, 2012.

[12] A. Sparavigna and R. Marazzato, "Mapping images with the coherence length diagrams," Computer Vision and Pattern
Recognition, International Journal of Software Engineering and Computing, vol. 1, pp. 53-57, 2009.

[13] G. W. Gray, Molecular Structure and the Properties of Liquid Crystals, Academic Press, New York, NY, USA, 1962.

[14] R. C. Gonzalez, R. E. Woods, and S. L. Eddins, Digital Image Processing using MATLAB, Dorling Kindersley, Delhi, India, 2004.

[15] M. Pietikäinen, A. Hadid, G. Zhao, and T. Ahonen, Computer Vision Using Local Binary Patterns, vol. 40 of Computational Imaging and Vision, Springer, London, UK, 2011.

[16] T. Maenpaa, Local Binary Pattern approach to texture analysis-Extensions and applications, Oulu Univeristy Press, Finland, 2003.

[17] T. Ojala, M. Pietikäinen, and T. Mäenpää, "Multi resolution gray-scale and rotation invariant texture classification with local binary patterns," IEEE Transactions on Pattern Analysis and Machine Intelligence, vol. 24, no. 7, pp. 971-987, 2002.

[18] T. Ojala, M. Pietikäinen, and D. Harwood, "Performance evaluation of textures measures with classification based on Kullback discrimination of distributions," in Proceedings of the International Conference on Pattern Recognition (ICPR '94), pp. 582-585, Jerusalem, Israel, 1994.

[19] LBP bibliography, http://www.cse.oulu.fi/CMV/LBP_Bibliography.

[20] F. Albregtsen, Texture Measures Computed from GLCMMatrices [Ph.D. thesis], Image Processing Laboratory, Department of Informatics, University of Oslo, 2008.

[21] M. Roumi, Implementing texture feature extraction algorithms on FPGA, computer engineering, mekelweg 4, 2628 CD [M.S. thesis], Delft University of Technology, The Netherlands, 2009.

[22] G. Saini, Texture Analysis of CT Scan Images [M.S. thesis], Electrical and Instrumentation Department, Thapar University, Patiala, India, 2008.

[23] R. Pavlis, Kansas USA edition of Micscape Magazine, Microscopy UK Front Page Published in the May 2006. 

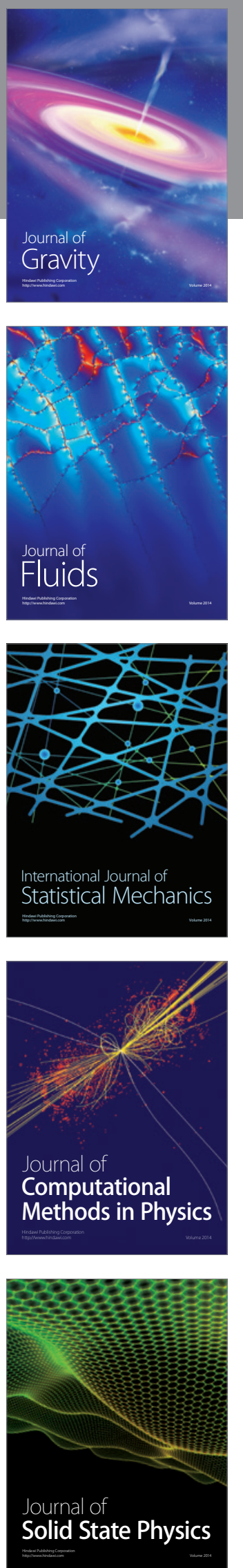

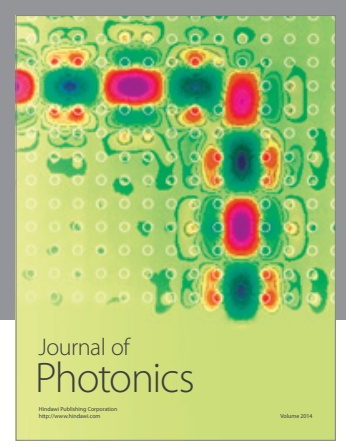

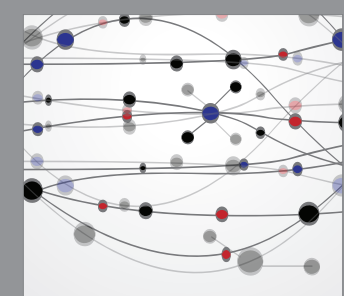

The Scientific World Journal
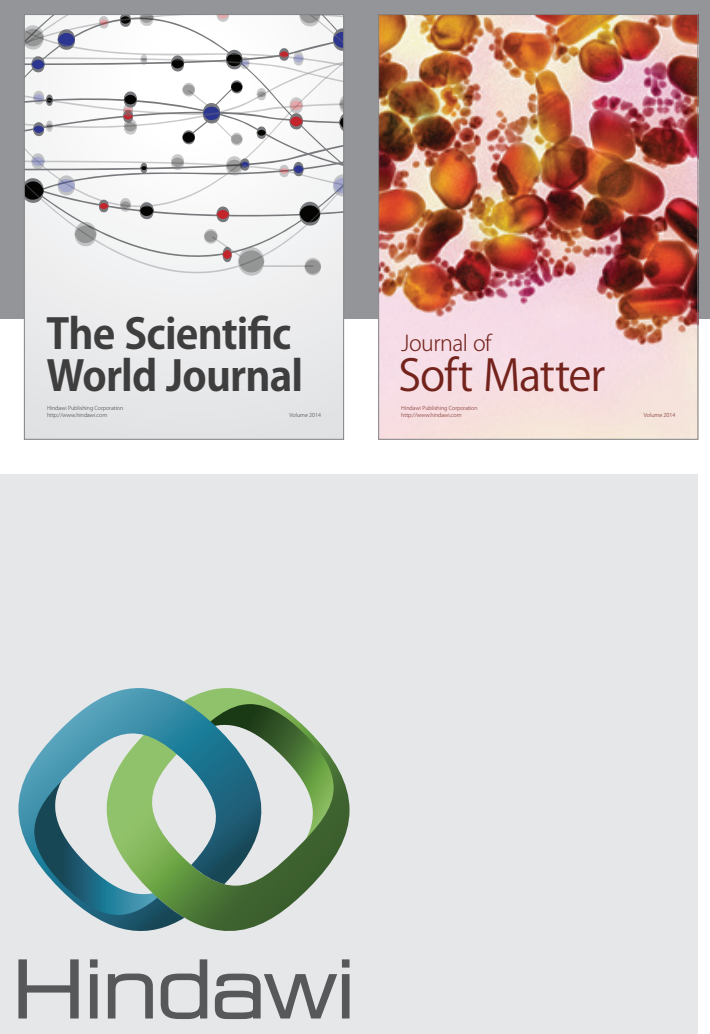

Submit your manuscripts at

http://www.hindawi.com
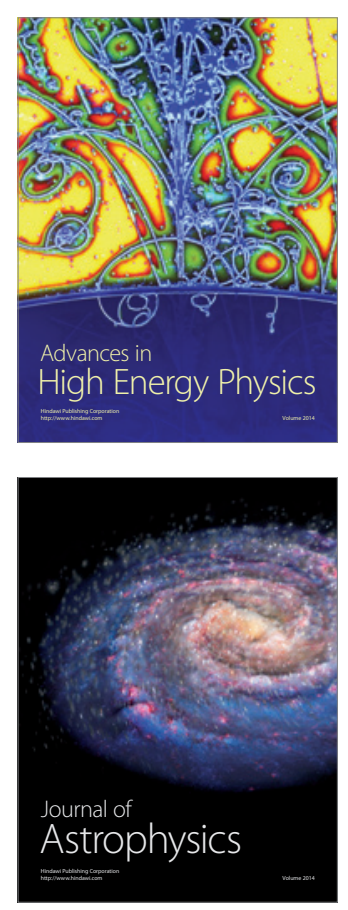
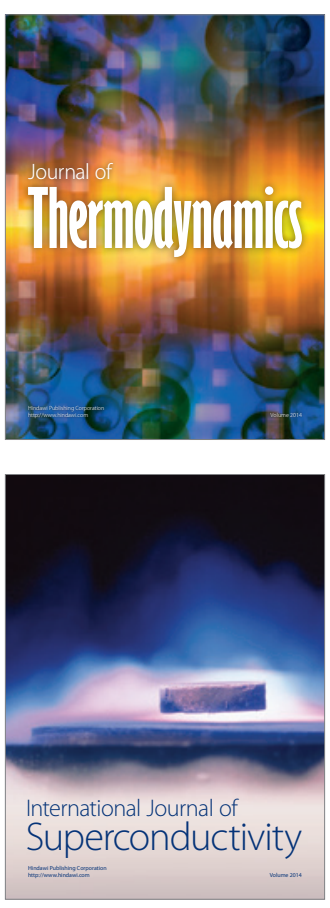
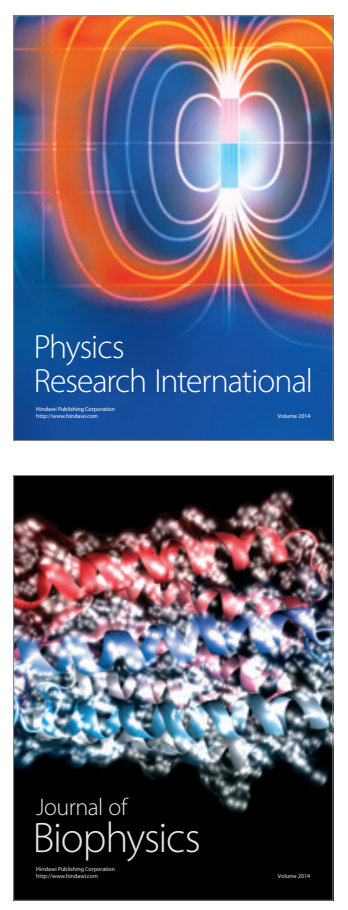
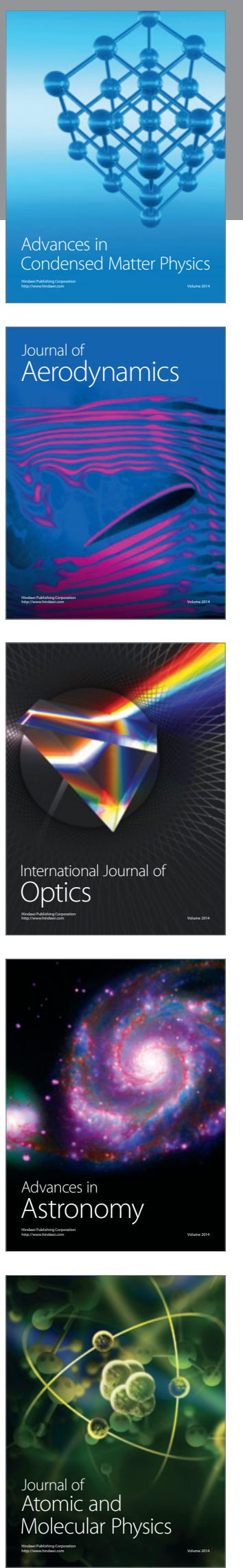